\title{
Corela
}

Cognition, représentation, langage

HS-2 | 2005

Le traitement lexicographique des noms propres

\section{Nom propre et lexicographie française}

\author{
Jean Louis Vaxelaire
}

\section{(2) OpenEdition}

Journals

Édition électronique

URL : http://journals.openedition.org/corela/1239

DOI : 10.4000/corela.1239

ISSN : 1638-573X

Éditeur

Cercle linguistique du Centre et de l'Ouest - CerLICO

Référence électronique

Jean Louis Vaxelaire, « Nom propre et lexicographie française », Corela [En ligne], HS-2 | 2005, mis en ligne le 02 décembre 2005, consulté le 02 avril 2021. URL : http://journals.openedition.org/corela/ 1239 ; DOI : https://doi.org/10.4000/corela.1239

Ce document a été généré automatiquement le 2 avril 2021.

\section{(c) (i) (2) (2)}

Corela - cognition, représentation, langage est mis à disposition selon les termes de la licence Creative Commons Attribution - Pas d'Utilisation Commerciale - Partage dans les Mêmes Conditions 4.0 International. 


\title{
Nom propre et lexicographie française
}

\author{
Jean Louis Vaxelaire
}

\section{Introduction}

1 La place du nom propre dans la lexicographie française semble bien définie puisqu'il existe des dictionnaires qui lui sont entièrement consacrés. Pourtant, un examen plus approfondi montre une situation nettement moins précise : tout d'abord, on recense des noms propres dans les dictionnaires de noms communs et des noms communs dans les dictionnaires de noms propres. Ensuite, les choix dans les nomenclatures des dictionnaires de noms propres sont particulièrement subjectifs et laissent la porte ouverte à la critique.

2 Cette étude va en premier lieu porter sur les dictionnaires de langue puis sur les dictionnaires de noms propres. Notre premier corpus est composé par la première partie du Petit Larousse Illustré(2000) (désormais PLI), le Nouveau Petit Robert (2000) (désormais PR1) et le Trésor de la langue française informatisé (TLFi) consulté en ligne en mars 2005. Le second comprend le Hachette des Noms propres de 1994 (désormais HNP), la dernière partie du Petit Larousse Illustré(2000) et le Petit Robert des noms propres (2000) (désormais PR2) 1 .

\section{Les noms propres dans les dictionnaires de langue}

3 Les travaux sur les caractéristiques des dictionnaires de langue face à celles des encyclopédies ne se comptent plus. Si l'on s'en tient à la doxa, le nom propre relève du second type d'ouvrage et ne peut donc apparaitre dans un dictionnaire classique. Ainsi, dans les homonymes d'un dictionnaire de langue, il n'y a pas de renvoi à Rubicon dans l'article rubicond ou à Paris dans pari.

4 On sait toutefois que le modèle théorique du dictionnaire de langue pur n'est pas autant mis en pratique que ne le prétendent ses partisans. Il existe ainsi des différences entre 
les dictionnaires américains et anglais, les premiers intégrant plus de noms propres, même s'ils peuvent eux aussi garder des réticences. Ainsi, le Webster's Third New International Dictionary of the English Language Unabridged décrit le nom propre uniquement dans l'article consacré à l'adjectif correspondant.

5 Dans les dictionnaires français, bien qu'il soit censé être absent, le nom propre peut servir à la construction des énoncés lexicographiques. On en retrouve finalement beaucoup dans les exemples : mont renvoie à Mont Blanc, Alpes, Monts du Cantal ou zouave à pont de l'Alma, Seine, Paris. Dans l'étude de Boulanger \& Cormier (1998: 104-107) qui porte sur le PLI et le PR1, on découvre respectivement des noms propres :

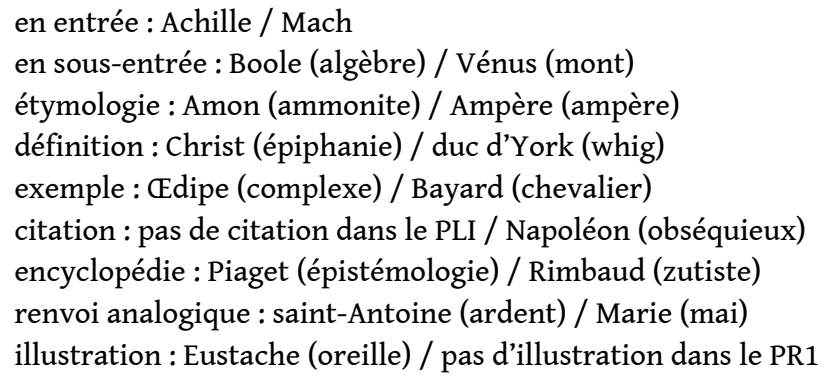

Le premier exemple tiré du PR1 est particulièrement intéressant. La marque "n.pr.» est exceptionnelle dans ce dictionnaire. Toutefois, Mach la reçoit alors qu'aussi bien dans nombre de Mach que dans Voler à Mach 2, il n'y a de nom propre : nombre de Mach est un nom commun (ou un terme) et Voler à Mach 2 une locution. Alors que les genres sont donnés, il n'apparaît pas ici (ce qui est d'une certaine manière logique puisqu'il n'y a ni un Mach ni une Mach). Dans le TLFi, Mach reçoit la marque "subs. masc.", et contrairement au PR1, il est noté que le nom s'écrit « avec ou sans majuscule ».

Un cas supplémentaire n'a pas été abordé par Boulanger \& Cormier, celui des différents sens au sein d'un même article. Ainsi, dans le sens 1 de apocalypse, on lit : «L'Apocalypse : dernier livre du Nouveau Testament, attribué par l'Église à saint Jean l'Évangéliste, riche en visions symboliques, prophétiques et eschatologiques. La bête, les quatre cavaliers de l'Apocalypse. PAR ANAL. Ouvrage eschatologique. Les apocalypses juives. » Si l'on observe la définition du PR2, on se rend compte qu'elle est seulement un peu plus développée que celle que nous venons de voir et ne comprend pas l'analogie. Si l'Apocalypse du PR2 est un nom propre, il en découle que celle du PR1 en est également un.

On retrouve le même phénomène avec le sens 1 de genèse: "Le premier livre de l'Ancien Testament qui contient le récit de la création. La Genèse. PAR EXT. $\rightarrow$ cosmogonie. » La Genèse en question est le titre d'un livre et relève en conséquence de la catégorie des noms propres.

9 En dehors des titres, l'exemple de sagittaire est intéressant. D'après le sens 1 du PR1, sagittaire est un terme d'astronomie dont la définition est : "Constellation zodiacale de l'hémisphère austral ("l'Archer").» Dans le sens B1 de ce mot, le TLFi ajoute la précision que ce terme s'écrit souvent avec une majuscule. La définition est proche de celle du PR1, quoique plus développée : "ASTRON. Constellation la plus australe par rapport à la Terre, en partie englobée dans la Voie lactée, que l'on représente dans les anciennes cartes du ciel sous la forme d'un centaure armé d'un arc. Un nuage important d'étoiles du Sagittaire devient alors visible, qui n'est sans doute pas le centre même de la galaxie, mais plutôt une partie du noyau de la galaxie (SCHATZMAN, 
Astrophys., 1963, p. 104).» Si l'on considère que les noms de planètes tels que Mercure ou Saturne sont des noms propres, pourquoi ne pas penser que les noms de constellations en sont également? Le PR2 partage cette opinion puisqu'il offre une entrée à Sagittaire ( La plus australe des constellations zodiacales: une moitié se trouve englobée dans la Voie lactée. »)

Le sens 2 de mage dans le PR1 évoque des personnes particulières : « SPÉCIALT. Les Mages : les personnages qui, selon l'Évangile, vinrent rendre hommage à l'Enfant Jésus. » À nouveau, le PR2 possède une entrée pour ce mot, même si elle ne fait que renvoyer à Balthazar, Gaspard et Melchior. À l'inverse, si le sens 2 de hexagone renvoie sans hésitation

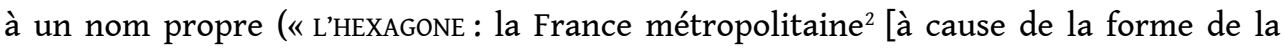
carte de France, qu'on peut inscrire dans un hexagone].»), il n'y a pas d'article Hexagone dans le PR2.

11 Un autre point n'a pas été relevé dans l'étude citée précédemment: la présence de synonymes. Par exemple, dans l'entrée empire du PR1, on lit : « MOD. L'Empire chérifien : le Maroc ». La consultation de ce dictionnaire fournit des exemples supplémentaires comme par exemple «MOD. L'X: l'École polytechnique», «PAR EXT. Paris-Plage: Le Touquet » et « Le Roi-Soleil : Louis XIV ».

12 La logique voudrait que le synonyme d'un nom propre (Louis XIV, le Maroc) soit luimême un nom propre. On pourra toujours nier ce statut pour certains cas mais d'autres sont incontestables : lorsque le Roi-Soleil est évoqué, les francophones savent qu'il s'agit de Louis XIV et non d'un autre souverain.

Dans d'autres exemples, on est confronté à un problème de définition. L'article Noël du PR1, possède une sous-entrée père Noël: "personnage imaginaire qui est censé descendre par la cheminée au cours de la nuit de Noël pour déposer des cadeaux dans les souliers des enfants. Croire au père Noël. » Il n'y pas d'entrée Noël dans le PR2, mais il est difficile de voir en quoi cette définition est différente de celle de Mickey Mouse: «Personnage de dessins animés, créé par Walt Disney. [...] ». Pourquoi le mot désignant un personnage imaginaire serait un nom commun alors que celui qui désigne un personnage de dessins animés serait un nom propre ? La distinction dépasserait dans ce cas les définitions classiques du nom propre et du nom commun.

14 Un autre exemple est extrêmement problématique, celui de Far West. L'article débute par la marque «n.m. inv. » et non «n.pr. » mais le nom est tout de même écrit avec des hauts de casse initiaux. Dans le corps, si l'exemple figuré « Débrouillez-vous, ici c'est le far west » contient un nom commun (le PR1 a d'ailleurs enlevé les majuscules), la première définition («territoire situé à l'ouest du Mississippi ») ressemble fortement à celle d'un nom propre.

Le problème est identique avec le Félibrige dans le TLFi, qui s'écrit avec une majuscule dans l'exemple. La définition fait elle aussi penser à un nom propre: «École, association littéraire fondée à l'initiative de Mistral et d'autres écrivains provençaux pour assurer la sauvegarde et la perpétuation culturelles de la langue provençale.» Félibrige réfère alors à une entité unique et peut prétendre être considéré en tant que nom propre. Ce nom fait également ressortir le manque de cohérence entre les deux dictionnaires Robert: le félibrige s'écrit sans majuscule dans le PR1 alors que la définition est typique de celle d'un nom propre ("École littéraire fondée en Provence (1854) par sept jeunes félibres. ») alors qu'il prend une majuscule dans le PR2. Il s'agit 
pour cet ouvrage d'un nom propre - le nom d'un mouvement littéraire - bien qu'il y ait eu plusieurs Félibriges en Provence et même un à Paris en 1879.

D'autres cas montrent des définitions dignes de noms propres :

PAMPA n.f. Vaste plaine d'Amérique du Sud, dont le climat et la végétation sont ceux de la steppe. Les gauchos de la pampa.

SAHEL n.m. 1. Région de collines littorales en Algérie et en Tunisie. [...] Les pays du Sahel : Mauritanie, Sénégal, Mali, Burkina-Faso, Niger, Tchad, Soudan. La famine menace les habitants du Sahel.

Si pampa est en bas de casse, ce n'est pas le cas de Sahel, pourtant les deux noms se retrouvent également dans les dictionnaires de noms propres. Les deux définitions de Sahel réapparaissent dans l'ordre inverse dans le PR2. La première concerne le Sahel subsaharien qui comprend la Mauritanie, le Sénégal, etc. La seconde est relative aux collines littorales d'Afrique du Nord, les rédacteurs nous parlent alors du Sahel d'Alger et des Sahels de Sousse et de Sfax. On notera le -s final de Sahels alors que, selon la doxa, les noms propres n'ont aucune flexion morphologique.

Il existe enfin un problème posé par les noms de produits :

Martini : «n.m.» «1. Vermouth produit par la firme Martini et Rossi. Du martini

blanc, rouge. Des Martinis. »

Les dictionnaires les définissent en tant que noms communs, bien qu'ils aient une majuscule initiale. D'un point de vue linguistique, ce sont effectivement des noms communs, malgré la volonté de certaines entreprises de présenter le nom de produit avec une majuscule et sans article (« versez Maïzena, délayez Maïzena »). Dans le TLFI, maïzena s'écrit sans majuscule dans les exemples et l'article ne laisse aucun doute sur le statut de ce type de nom.

Bien que censé être un dictionnaire de langue (ce qu'annonce sa préface), le PR1 contient différents types de noms propres: des anthroponymes, mais aussi des toponymes, des titres d'œuvres (L'Avare de Molière ou Les misérables de Hugo aux entrées correspondantes), ou encore des noms de bâtiments comme la Maison Blanche dans l'article maison ${ }^{3}$. Toutefois, ces noms propres ne sont pas utilisés en tant qu'éléments linguistiques mais en tant qu'illustrations, compléments encyclopédiques ou caution.

\section{Les noms communs dans les dictionnaires de noms propres}

21 La présence de noms communs à la nomenclature du PR2 n'est pas un secret ${ }^{4}$. D'après la préface d'Alain Rey, ils servent à assurer "une homogénéité de traitement à des faits historiques comme la résistance ou la collaboration, à des mouvements artistiques ou littéraires comme le baroque ou le naturalisme, à des doctrines philosophiques, religieuses, politiques comme l'anarchisme ou le bouddhisme, par rapport à ceux qui sont désignés par des noms propres" (2000: XIX). On aperçoit différents types de noms communs tels que des noms de religions (jaïnisme), de mouvements artistiques ou littéraires (hyperréalisme), de philosophies (cartésianisme), de mouvements politiques (communisme) ou encore de groupes (carlistes). Ces entrées sont distinguées physiquement des noms propres par un encadrement. 
22 Parmi nos dictionnaires de noms propres, le HNP est l'ouvrage ayant proportionnellement le plus de noms communs à sa nomenclature. Dans la note de l'éditeur, on apprend que ces noms communs renvoient «à des thèmes ou des notions entrant dans le champ de la connaissance littéraire ou artistique » mais ils ne sont pas différenciés des noms propres dans le texte. Le choix est varié : il y a principalement des genres artistiques (Action painting, roman breton, constructivisme, haiku) mais l'on note des types très divers de noms communs (par exemple carnets, centaures, croisade, esthétique, géographie, sénat, voyage, zodiaque, etc.). Au niveau du contenu, on peut les distinguer des articles carnet, esthétique, zodiaque, etc. dans le PR1 ou le TLFi puisqu'ils semblent comparativement tirés d'une encyclopédie.

Les deux ouvrages défendent l'idée d'une cohérence thématique entre noms propres et noms communs présents, mais si l'on pousse cette notion à l'extrême, n'importe quel nom commun peut avoir un lien avec un nom propre et pourrait trouver sa place dans un dictionnaire de noms propres.

L'idée de regroupement thématique est caractéristique de l'encyclopédie mais il serait excessif de considérer que les dictionnaires de noms propres sont des encyclopédies qui cachent leur nom sur la base de cet unique élément. Les nomenclatures conservent un ordre alphabétique (bien que ce point soit discutable comme nous le verrons dans le chapitre 3.1.) et ne possèdent pas a priori de sections thématiques sur un sujet particulier dans le corps du texte. Il est donc possible de considérer que ce sont des considérations pratiques qui ont amené les concepteurs du PR2 et du HNP à inclure quelques noms communs, afin qu'ils complètent les noms propres dans un même volume. Ainsi, la lecture de l'article Ménippe permet d'apprendre qu'il s'agit d'un "philosophe et poète grec de l'école cynique [...]» et l'article cyniques apporte d'autres informations sur les membres de cette école et leurs préceptes. À l'inverse, ce sont des considérations purement encyclopédiques qui amènent à la rédaction de l'article encadré - correspondant donc à un nom commun - docteurs de l'Église du PR2. La définition est brève («Titre décerné par l'Église à des théologiens ou auteurs spirituels dont l'enseignement est reconnu éminent.»), suivie d'une liste de noms de docteurs ayant chacun un article correspondant. Pourtant, lorsqu'on lit les articles consacrés à Anselme et Albert le Grand, il n'est fait aucune allusion à leur statut de docteur, ce qui annule tout renvoi vers docteurs de l'Église. La visée encyclopédique des articles autobiographie, chant, fragment, lai, oratorio ou sextuor ${ }^{5} \mathrm{du}$ HNP est encore plus transparente. Il est pratiquement inutile d'ajouter qu'ils ne renvoient à aucun nom propre.

25 La présence de noms communs à la nomenclature des dictionnaires de noms propres entraîne naturellement des questions sur la différenciation entre noms communs et noms propres et sur leur définition. Aucun de ces dictionnaires ne propose en préface ou en quatrième de couverture une définition du nom propre, celle-ci étant sans doute par trop évidente. L'étude des articles introduit pourtant un doute quant à cette définition. Dans le PR2, Galles (prince de) décrit par «titre porté par le fils aîné du roi ou de la reine d'Angleterre depuis 1301 » est considéré en tant que nom propre alors que panchen lama, qui est également un titre («titre du supérieur du monastère de Tashilumpo [...]»), est traité en tant que nom commun. Il y a certes une distinction au niveau de la majuscule initiale, mais cet argument est faible ${ }^{6}$. Si l'on observe maintenant des articles de noms de titres dans le PR1 (par exemple « NÉGUS: Titre porté par les souverains éthiopiens. » et « RHINGRAVE : Titre porté par les princes allemands de 
la région rhénane.»), il appert que leur définition est similaire à celle de Galles (prince de). Quelle particularité fait de cette séquence un nom propre alors que les autres noms de titres sont des noms communs? Cette question demeurera sans réponse, bien qu'on puisse imaginer que la présence du nom propre Galles a eu une influence ${ }^{7}$.

À l'inverse, kremlin et beat generation sont classés en tant que noms communs dans le PR2 alors qu'ils semblent être pour diverses personnes des noms propres. Si l'on reprend l'exemple du Félibrige, il est décrit en tant que «mouvement littéraire pour la renaissance de la langue d'oc [...]» alors que beat generation est un "mouvement littéraire et social qui se développa aux États-Unis entre 1950 et 1960 [...] ». On peut à nouveau être sceptique devant la similarité des définitions entre le nom propre Félibrige et le nom commun beat generation.

Ce que démontrent ces exemples, c'est que le fait que les dictionnaires ne présentent pas de définition explicite du nom propre entraîne un flou dommageable. Les rédacteurs des dictionnaires partagent-ils tous la même définition de ce qu'est un nom propre ? Cette question demeurera sans réponse.

\section{Le traitement des noms propres dans les dictionnaires de noms propres}

Bien qu'il y ait deux modèles de dictionnaires sur le marché français, ils comportent chacun des noms qui devraient apparaitre dans l'autre type d'ouvrage. Si la distinction n'est pas établie entre d'un côté les noms communs (et les verbes, adjectifs, adverbes, etc.) et de l'autre les noms propres, c'est que d'autres critères d'inclusion et d'exclusion entrent en ligne de compte.

\subsection{Ordre des entrées}

Puisque le dictionnaire de noms propres n'est pas une encyclopédie thématique, le classement des entrées se fait par ordre alphabétique. Alain Rey annonce d'ailleurs dans sa préface du PR2 que «l'ordre alphabétique est ici appliqué à la totalité de l'entrée ». Cependant, dans le cas des homonymes, le classement alphabétique tend à être supplanté par d'autres choix. Ainsi, le PR2 et le HNP ne classent pas les différents Martin ou Smith selon l'alphabet mais selon l'ordre chronologique : Pierre Martin précède Archer Martin dans la nomenclature car il est né le premier. Le PLI est le seul à ne pas se préoccuper des dates de naissance : l'entrée Frank Martin est donc placée avant l'entrée Nicolas Martin. Toutefois, dans l'article Marx Brothers, les frères Marx sont rangés selon leur date de naissance et non leur prénom. Le même fonctionnement s'applique aux frères Adam ou aux père et fils Johnson ${ }^{8}$.

\subsection{Poids de l'ontologie}

Les questions d'ordre ontologique sont depuis toujours prégnantes dans le débat sur les noms propres, mais il est tout de même surprenant de les voir transparaître de manière si nette dans des dictionnaires. Que ce soit par le classement alphabétique ou la typographie, certains ouvrages insistent sur la différence entre anthroponymes et noms de personnages de fiction. 
31 Les noms issus de la littérature sont rangés dans le PLI comme les autres noms de personne selon leur patronyme: Arsène Lupin est dans les L, Sherlock Holmes dans les H, James Bond dans les B, etc. Des noms comme Sancho Pança et Peter Pan viennent pourtant rompre cette harmonie et sont rangés selon leur prénom.

Le PR2 a choisi l'option inverse du PLI : Arsène Lupin, Fabrice del Dongo, James Bond, Jean Valjean, Julien Sorel, Peter Pan, Sancho Pança et Sherlock Holmes sont rangés selon leur prénom. D'autres comme Eugène de Rastignac ou Joseph Prudhomme sont classés par rapport au patronyme, mais leur prénom n'est peut-être pas assez célèbre pour permettre un autre choix. Ce classement se répercute sur d'autres noms fictifs tel que Oncle Sam qui est classé dans les O dans le PR2 alors qu'il est dans les S pour le HNP ${ }^{9}$ et le PLI.

Ces options sont plus que contestables: le lecteur se retrouve dans une situation où Sherlock Holmes est classé selon son prénom dans le PR2 et le HNP et où Arthur Holmes l'est par rapport à son patronyme, alors que rien ne l'indique au niveau du péridictionnaire. On peut simplement se demander s'il entre dans les attributions des lexicographes de se prononcer sur la réalité de tel ou tel référent.

Les choix typographiques influencés par des considérations ontologiques ne sont, eux non plus, pas explicités dans les préfaces. Ainsi, seule une étude attentive permet de noter la distinction qu'établit graphiquement le PR2 entre lieu imaginaire (Liliput) et lieu réel (LIMBOURG). Le PLI en fait de même avec BOURBAKI (Charles) et Bourbaki (Nicolas). La différence faite par le PLI entre noms de la mythologie en majuscules (PAN) et noms de personnages en minuscules (Pantalon) est plus étonnante; les dieux seraient-ils plus réels que les personnages de fiction pour les éditeurs?

Que ce soit au niveau de l'ordre des entrées ou dans ces choix typographiques, les critères de sélection ne sont nullement linguistiques, seul le référent est pris en compte, ce qui est problématique dans le cadre d'un dictionnaire.

\subsection{Morphèmes}

En tant qu'unités linguistiques minimales, les morphèmes ont fait leur apparition dans des dictionnaires de langue tel que le PR1. Le HNP propose deux entrées pour des morphèmes faisant partie de patronymes: $O$ ' qui est défini comme une "particule précédant les noms propres irlandais, qui signifie "fils de" " et Mac, un "mot celtique ("fils"), qui entre comme préfixe dans des noms propres d'origine irlandaise ou écossaise ». Ces éléments, rangés de manière alphabétique dans le HNP, sont absents des autres dictionnaires. Leurs auteurs peuvent certainement arguer qu'il ne s'agit pas de noms propres, ce qui est vrai. Il me semble néanmoins qu'il s'agit là d'une information de nature linguistique et, par conséquent, très utile. Il serait certainement profitable d'y ajouter par exemple les suffixes slaves : une terminaison en -ska indique un nom polonais féminin (la version masculine est en -ski) ; -ovic est un suffixe venant du serbo-croate, etc.

37 La présence de ces morphèmes serait utile lorsque l'on est confronté au type de néologisme que comporte cet extrait :

Les mouvements d'actions suspects qui ont précédé l'annonce de la transaction pourraient contrarier le sauveur de Chelsea, que le quotidien populaire The Sun a rebaptisé « Chelski ». (Le Monde, 04/07/03) 
Le sauveur en question est un homme d'affaires russe, ce qui explique pourquoi le néologisme Chelski est parfaitement compréhensible: le morphème -ski véhicule un sens aisément identifiable pour les lecteurs du Sun.

Aucun référent n'est lié à ces morphèmes et le dictionnaire de noms propres tel qu'il est actuellement envisagé nécessite la présence de référents bien identifiables. Puisque la plus petite unité du dictionnaire de noms propres est la lexie et non le morphème, il n'y a pas non plus de morphèmes productifs tel que -gate que l'on retrouve très couramment dans la presse (Monicagate, Angolagate, etc.).

\subsection{Informations sur le signe}

40

On est en droit d'attendre d'un dictionnaire des informations sur le signe linguistique qu'est le nom propre. Cette dimension semble pourtant secondaire pour les rédacteurs.

Ainsi, le HNP ne propose aucune prononciation et les deux autres de manière très rare. En fait, le PR2 annonce qu'il ne donnera la prononciation que de noms français posant un problème par rapport aux normes, sans pour autant présenter d'exemples. Il est facile de les trouver car il s'agit en fait de cas classiques (Bourg-en-Bresse, Broglie, Chamonix, Montréal, St-Saëns, St-Tropez, Saône, Schlucht). Toutefois, contrairement à ce qui est annoncé dans la préface, on aperçoit de très rares noms étrangers (par exemple Wehrmacht). Le cas de Quasimodo est particulièrement intéressant car le PR2 donne la prononciation du nom du personnage (tiré d'un roman français, donc d'une certaine manière naturalisé) mais pas celle de l'Italien Salvatore Quasimodo (qui serait différente si l'on essayait de reproduire la prononciation italienne). Le PLI a opté pour le même choix et présente sensiblement la même sélection de noms français et propose exceptionnellement des noms étrangers (Charybde, Chéronée, Milosz, Munich, Quirinal).

Cette option est, comme l'avait noté M.-N. Gary-Prieur (1991: 9), problématique: comment un francophone doit-il prononcer des noms tels que Utrecht ou Drzic s'il ne parle ni le néerlandais, ni le serbo-croate? Les exemples pourraient être multipliés : ainsi, doit-on débuter Phnom Penh et Phuket avec un son [p] ou [f] ? Se limiter à la prononciation des noms français n'est effectivement pas satisfaisant. de prononciation: STO est un sigle et non un acronyme, ce que pourrait laisser supposer la consultation du dictionnaire.

Deux des cinq dictionnaires sur lesquels a travaillé A. Lecomte-Hilmy (1989) se retrouvent dans notre panel - le PR2 et le PLI -, les autres étant le Robert - Dictionnaire universel des noms propres, le Pluridictionnaire Larousse et le Grand Dictionnaire encyclopédique Larousse. Pour elle (1989: 10), les deux Robert sont les seuls à donner le genre proprement dit, sauf pour les noms de personnes, de personnages et d'œuvres. Des améliorations ont été apportées au PLI et l'on retrouve dans l'édition de 2000 des informations concernant le genre bien que certains noms aient été oubliés (Scapa Flow, Shijing). À l'inverse, le HNP ne donne jamais d'indications sur le genre, mais le fournit par exemple pour les noms de rivières et de fleuves grâce au déterminant : Meurthe (la), Garonne (la). Cette procédure n'est pas exclusive, on ne saura pas par exemple le genre des rivières Ili, Ill, Ille et Iller. 
Aucun de ces dictionnaires n'est prolixe en matière d'information sur les déterminants. A. Lecomte-Hilmy (ibid. : 14) a ainsi noté que Rhune (la) est le seul nom de montagne qui apparaît avec un article dans le PLI ${ }^{10}$ et le PR2.

e semble être de ne pas donner de déterminant, sauf lorsque celui-ci est indissociable de l'autre partie du nom propre (par exemple Déjeuner sur l'herbe [le]). Cette option entraîne des résultats peu souhaitables : Action catholique Ø est suivi dans le PLI par Action française ( $\left(l^{\prime}\right)$, ce qui laisse penser que le premier ne s'emploie jamais avec un $\operatorname{article}^{11}$. L'absence totale d'indications est également, d'après A. Lecomte-Hilmy (ibid. : 22), préjudiciable dans des cas comme Israël qui s'utilise sans article, ou TerreNeuve qui est précédé de à et non de en. L'exemple d'Israël n'est pas judicieux car le nom sera employé dans le corps de l'article sans déterminant, la comparaison entre RTBF et $R T L$, deux noms qui se suivent dans le PLI est plus parlante : les articles étant plus brefs, les rédacteurs font appels à des pronoms anaphoriques et le lecteur ne saura donc pas que RTBF s'emploie avec un déterminant (en l'occurrence la), ce qui n'est pas le cas de RTL.

7 Parmi les informations sur le signe, les lexicographes citent, aux côtés de la prononciation et des constructions syntaxiques, les locutions. Il existe diverses locutions comprenant des noms propres et il me semblait instructif de recenser, à partir d'un corpus composé des plus courantes, celles qui étaient définies dans les dictionnaires de noms propres.

8 L'examen fait apparaître que la présence ou l'absence des locutions semble liée à la notoriété des référents. Byzance, Rabelais ou le Pérou sont plus saillants dans notre culture que Artaban ou Jarnac et pourtant les locutions correspondant à ces trois premiers exemples (C'est Byzance, Le quart d'heure de Rabelais et Ce n'est pas le Pérou) ne sont pas traitées contrairement aux autres (Fier comme Artaban et Aller à Canossa). Doiton en conclure que la locution doit correspondre à un nom propre peu notoire pour être définie ? Il y a effectivement peu à dire sur Canossa, on peut à l'inverse donner des quantités d'informations sur Rabelais.

9 Si des locutions telles que riche comme Crésus et talon d'Achille sont généralement définies dans les dictionnaires de noms communs, ce n'est pas le cas d'autres comme C'est Byzance, où le nom propre porte tout le signifié de la locution. En raison des partis pris des dictionnaires de noms propres et de leur absence peu surprenante des dictionnaires de langue, il est donc impossible d'obtenir la définition de cette locution et de Ce n'est pas le Pérou.

Les relations de synonymie sont pratiquement inexistantes dans les dictionnaires de noms propres. Il y a certes des renvois, mais ils sont uniquement thématiques. Lorsque le PR1 définit Hexagone en tant que synonyme de la France, le PR2 ne cite pas ce nom et ne lui offre aucune entrée. J'ai parlé dans la première partie de l'X et du Roi-Soleil ; ils n'apparaissent pas plus à la nomenclature du PR2. Si certains chercheurs refusent de parler de synonymie stricto sensu au sujet des noms propres, il est tout de même incontestable que certains ont des surnoms. On recense quelques surnoms au sein des articles (Satchmo / Louis Armstrong; The King / Elvis Presley; Papa Doc / François Duvalier ${ }^{12}$ ), mais il n'y a jamais d'entrées Satchmo, The King ou Papa Doc. Le seul surnom ayant une entrée propre dans les trois dictionnaires est Marianne, mais il n'y a pas non plus de renvoi vers Marianne dans les articles France ou République. 
51 Enfin, la présence de tableaux et de cartes dans les dictionnaires de noms propres indiquent une nette visée encyclopédique. La portée linguistique des tableaux de fin d'ouvrage tels que les "monnaies» dans le HNP, la "numérotation administrative " dans le PR2 et la «liste des lauréats des prix Nobel» dans le PLI ont une portée linguistique très faible.

52 Comme le signale lucidement A. Lecomte-Hilmy, « dans leur avis aux lecteurs (PLI) et leur avant-propos (Pluri et GDEL), les auteurs n'envisagent pas qu'à l'égard du nom propre le lecteur soit à la recherche d'une information autre que de nature encyclopédique » (1989: 29). Le dictionnaire de noms propres est en effet un genre ayant une portée heuristique très limitée puisque les informations sur le signe sont excessivement limitées. On ne peut qu'être circonspect en voyant le traitement de Sotheby's dans le HNP : on ne saura pas comment ce nom se prononce, quel est son fonctionnement syntaxique en français (s'emploie-t-il avec ou sans déterminant), on apprendra par contre que cet établissement est situé au 34 New Bond Street W1 à Londres.

\section{Choix des entrées}

Adopter une approche moins encyclopédique permettrait d'alléger la nomenclature des noms communs déjà présents dans les dictionnaires de langue. Il s'agit donc de considérer quels éléments pourraient remplir les vides laissés.

Alain Rey écrit dans la préface du PR2 qu'« un répertoire des noms propres les plus nécessaires repose sur la notion de notoriété ». Toutefois, l'idée de notoriété qui soustend la construction du PR2 n'est pas exactement celle qui est définie dans les dictionnaires ${ }^{13}$. En effet, il importe que les anthroponymes soient tirés de la culture savante. Ce choix est conscient puisque Rey oppose le manque de notoriété de scientifiques importants à la "gloire peu glorieuse » de chanteurs à succès et d'hommes politiques scandaleux. Ainsi, on ne trouvera Claude François dans aucun dictionnaire et si Michael Jackson est depuis peu dans le PLI ${ }^{14}$, il n'est pas encore recensé dans les autres ouvrages.

J. Rey-Debove (1971 : 31) estime qu'il existe deux types de hiérarchisation :

- la place dans les taxinomies (plante rare vs rose) ou à l'intérieur d'un système socioculturel,

- la fréquence des occurrences dans le discours.

Le traitement des noms propres ne dépend que du premier puisque, d'après J. ReyDebove, "c'est l'importance d'une personne, d'un lieu, d'un événement et non la fréquence des noms qui les désignent, qui structure une nomenclature de noms propres. Ce fait est en accord avec l'afflux des noms propres dans les Encyclopédies (ensemble de choses-nommées) et leur rejet des dictionnaires qui répertorient un ensemble de signes-nommants" (ibid.). Ce parti pris référentialiste n'est pas défendable. Par quelle opération divine les noms propres devraient-ils être classés dans une structure plus ou moins taxinomique et non être comptabilisés en tant qu'occurrences? Il est évident que dans un corpus de textes consacrés à la musique, le nom Mozart apparaîtra plus souvent que celui de Xenakis (peut-être même dans certains textes consacrés à la musique contemporaine), mais on verra aussi des noms d'absents des dictionnaires tels que Michael Jackson ou Claude François. 
57 En tant qu'outil, le dictionnaire peut-il ainsi se permettre d'omettre des noms parce que ses éditeurs jugent que la personne qui le porte n'est pas digne de tant d'honneurs? Tout le monde aura probablement oublié Claude François dans quelques décennies, mais son nom apparaît aujourd'hui dans plus de textes que celui de Paul Gavarni ou de HarryMartinson, qui sont pourtant présents dans les dictionnaires. La fréquence nous semble un critère préférable dans un dictionnaire à une appréciation subjective et idéologiquement référentialiste.

Comme nous avons pu le voir avec les différences typographiques, les dictionnaires traitent différemment les noms issus de la fiction. Les autres types de noms fictifs sont quant à eux souvent absents : le Père Noël est présent dans l'article Noël du HNP mais ne figure ni dans le PR2, ni dans le PLI ${ }^{15}$. Des noms imaginaires comme celui de Vulcain (la planète que Le Verrier pensait avoir découvert grâce à de savants calculs et non celle du Docteur Spock qui relève des noms de fiction littéraire) sont absents de tous les dictionnaires et il en est de même pour ce que nous appellerons pour plus de commodité des «faux noms propres» (Trifouilly-les-Oies, Pétaouschnok, Madame Irma, etc.). Les dictionnaires semblent requérir un référent précis, le fait qu'il soit réel ou fictif joue un rôle, mais est tout de même secondaire. Un nom tel que Dupont n'est jamais défini en tant que nom français archétypique (ou comme l'équivalent français de Smith), mais est approché en tant que patronyme d'une personne bénéficiant d'une certaine célébrité (p. ex. Pierre Dupont ou Gabriel Dupont).

Une approche qui ne serait pas purement référentialiste permettrait d'inclure ces noms - qui sont absents de tous les types de dictionnaires -, ainsi que les morphèmes dont j'ai précédemment parlé.

\section{Conclusion}

60 Les noms propres ne sont pas traités comme les autres classes de mots dans les dictionnaires. Dans les dictionnaires de noms communs, ils sont généralement employés dans les exemples, en tant qu'éléments encyclopédiques, alors que les définitions lexicales sont possibles (les exemples de Far-West, Sagittaire cités plus haut). Dans les dictionnaires de noms propres, le linguistique est réduit au strict minimum, les noms ne sont jugés qu'en tant qu'entités encyclopédiques, généralement hors de tout contexte ${ }^{16}$.

61 L'intégration dans l'un ou l'autre de ces dictionnaires se fait donc en réalité sur une opposition encyclopédie vs langue et non sur une distinction nom propre vs nom commun. Les choix découlent alors de critères subjectifs - qui ne peuvent satisfaire que des personnes désirant consulter une encyclopédie - et contestables si l'on juge que les noms propres relèvent de la langue. Le dictionnaire de noms propres, tel qu'il existe en France, n'a qu'une valeur faible en tant qu'outil pour les non-francophones ou les traducteurs ${ }^{17}$.

62 Il est habituel dans les travaux métalexicographiques de s'étonner des absences de tel ou tel nom dans les dictionnaires (par exemple des métonymies aussi courantes actuellement que Place Beauvau et surtout Quai d'Orsay), mais il est évident que les contraintes éditoriales des dictionnaires-papier invitent les lexicographes à mûrement réfléchir avant de faire entrer un nouveau nom. C'est pour cette raison que le dictionnaire électronique est une solution pour le traitement du nom propre et de ses 
dérivés, en particulier pour les adjectifs qui pourraient être rattachés par des liens hypertextes au nom propre, et pour les antonomases contemporaines (un Rambo, un Poulidor, etc.) qui pourraient enfin être accueillies dans les dictionnaires, mais il est tout d'abord nécessaire de repenser notre conception des noms propres.

\section{BIBLIOGRAPHIE}

Boulanger Jean-Claude \& Cormier Monique C. (1998). « Les noms propres dans l'espace dictionnairique », in G. Ruffino (éd.), Vol. III, Lessicologia e semantica delle lingue romanze, Tübingen, Max Niemeyer, p. 99-110.

Lecomte-Hilmy Anne - 1989, « Du statut linguistique des noms propres dans cinq dictionnaires français ", Cahiers de lexicologie, Vol. 54, $n^{\circ} 1$, p. 8-32.

Gary-Prieur Marie-Noëlle (1991). « Le nom propre constitue-t-il une catégorie linguistique », Langue française, $\mathrm{n}^{\circ}$ 92, p. 4-25.

Rey-Debove Josette (1971). Étude linguistique et sémiotique des dictionnaires français contemporains, La Haye-Paris, Mouton, 329 p.

Vaxelaire Jean Louis (2005). Les noms propres - Une analyse lexicologique et historique, Paris, Honoré Champion, $952 \mathrm{p}$.

\section{NOTES}

1. Les variations au niveau de la nomenclature (25000 entrées pour le HNP contre 40000 pour le PR2) ou des années d'édition sont secondaires puisque le but principal de ce travail est d'étudier la structure de ces dictionnaires et leur approche des noms propres qui, elles, évoluent très lentement.

2. Il s'agit du sens 3 du TLFi, illustré par une citation de R. Beauvais : « Il y a environ deux mille ans, la France c'était la Gaule. Pendant des siècles, la France a été la France : aujourd'hui la France est encore la France, mais on l'appelle l'Hexagone. »

3. «L'élimination des noms propres dans le comptage, afin d'écarter les entrées purement encyclopédiques qui n'apparaissent pas dans les dictionnaires de langue, est plus délicate qu'on ne le pense généralement : le passage du nom propre au nom commun est aussi un continuum. Les dictionnaires de langue enregistrent souvent à leur insu un nombre non négligeable de noms propres (ex. C.G.T.). » (Rey-Debove, 1971: 63).

4. Le cas du PLI est particulier puisque noms propres et noms communs sont regroupés dans un même volume, ce qui limite naturellement la pertinence de l'inclusion de noms communs dans la partie noms propres.

5. On recense près d'une centaine de noms communs dans cet ouvrage.

6. Des occurrences de Panchen Lama sont visibles dans certains textes.

7. Le morphème rhin de Rhingrave renvoie lui aussi à un nom propre, le fleuve éponyme, mais il est probablement moins transparent que Galles. 
8. Il semblerait que, plus on acquiert une célébrité qui ne doit rien à sa famille, plus on a droit à une entrée séparée : contrairement à Daniel, Daniel J. et Pierre-Marc J. Johnson, James et John Stuart Mill ainsi que Auguste et Jean Renoir ne sont pas rangés sous la même entrée dans le PLI.

9. Toutefois, pourquoi le Sapeur Camember est-il dans les S si Oncle Sam est rangé selon la seconde partie du nom?

10. Ce nom a disparu de l'édition de 2000.

11. Ou dans le PR2, l'article Beatles (les) est suivi plus loin de l'article Rolling Stones Ø.

12. De nombreux autres qui semblent aussi saillants dans notre culture sont absents (par exemple Baby Doc / Jean-Claude Duvalier et Belle province / Québec). Le bilan est encore moins satisfaisant dans le PLI.

13. Pour le PR1 : «Caractère de ce qui est notoire ; le fait d'être connu d'une manière certaine et générale » ou « Fait d'être connu avantageusement ».

14. Ce nom n'apparaissait toujours pas dans l'édition de 1998.

15. On le retrouve dans les parties noms communs (cf. chapitre I).

16. Deux conceptions s'affrontent : une ligne référentialiste, depuis toujours dominante puisque de tradition logico-grammaticale, qui considère que le nom propre ne relève pas de la langue et une ligne linguistique qui juge que les noms propres sont avant tout des lexèmes, et par conséquent composés de morphèmes, avec un genre et un comportement syntaxique particulier. Le contexte est inutile dans la première et essentiel dans la seconde (Vaxelaire, 2005).

17. Il suffit pour s'en persuader d'observer les difficultés de traduction posées par les noms de la culture populaire dans les romans contemporains.

\section{RÉSUMÉS}

L'observation du traitement des noms propres dans les dictionnaires de langue et de noms propres permet d'obtenir des conclusions nettes sur l'approche principalement encyclopédique que ces ouvrages ont adopté. Le déficit linguistique des dictionnaires de noms propres peut cependant être corrigé en privilégiant comme critère de sélection la fréquence des occurrences et en donnant des informations sur le signe linguistique qu'est le nom propre.

Examining how proper nouns are treated in general language dictionaries and proper name dictionaries throws light on the mainly encyclopaedic approach that these works have adopted. However, the linguistic deficit of proper name dictionaries can be corrected by weighting frequency of the occurrences as criterion of selection and by giving information on the proper noun as a linguistic sign.

\section{INDEX}

Mots-clés : noms propres, lexicographie, dictionnaire, langue française 
AUTEUR

JEAN LOUIS VAXELAIRE

CIEL, Université Paris 7 